\title{
OPEN Polyaza functionalized graphene oxide nanomaterial based sensor for Escherichia coli detection in water matrices
}

\author{
Lina Rose ${ }^{1}$, X. Anitha Mary², I. Johnson ${ }^{3}$, Ganesh Srinivasan ${ }^{4}$, Lakshmi Priya $^{4}$ \& \\ Jebasingh Bhagavathsingh $\mathbb{1}^{4 \otimes}$
}

Water quality is widely discussed owing to its significance in public health due to the inability to access clean water. Waterborne diseases account for the presence of pathogens like Escherichia coli $(E$. coli $)$ in drinking water in the environmental community. Owing to the rapid increase of such bacterial microorganisms, a cost-effective sensor setup has been developed. Herein, we demonstrate the amine-functionalized graphene oxide $(f G O)$ based 2D nanomaterial used to graft $E$. coli on its surface. The comparative analysis of the deposition of nanosheets on the glass substrate and PDMS was executed. The impedance variations of GO-based nanosensor at various concentrations of $E$. coli were performed and their potential difference was recorded. It was observed that the impedance changes inversely with the bacterial concentrations and was fed to the Arduino microcontroller. The experimental setup was standardized for the range of $0.01 \mathrm{~Hz}$ to $100 \mathrm{kHz}$. The obtained analog data was programmed with a microcontroller and the bacterial concentration in colony-forming units was displayed. The real-time analysis showsthe low-level detection of $E$. coli in aquatic environments. Experiments were conducted using the developed nanosensor to test the efficiency in complex water matrices and whose behavior changes with various physical, chemical, and environmental factors.

Nanomaterial-enabled sensors have paved the way for the development of high efficient smart devices as sustainable systems in the areas of food, water, health, and the environment ${ }^{1}$. The technology of nanosensors has been a promising tool in the area of bacterial detection and the pandemic agents as the major contaminant in the aquatic environment. Recent studies show that the mortality rate in children under is mainly due to diarrhea and pneumonia as the water-borne disease ${ }^{2}$. These epidemics are considered the most common cause ofthe high mortality rate among children. As quoted in the literature, the unclean habitat in India makes the country a predominant victim among other countries. The prevalent source of bacterial diarrhea includes the infections caused by Shigella species, E. coli and Cholera toxins, and salmonella species ${ }^{3}$. The WHO has illustrated the waterborne contaminants and their ill effects, in which the most common is Escherichia coli ${ }^{4}$. As per the reports, E. coli is a pathogen with a high risk of health-related issues, which is more persistent in water, less resistant to chlorine, and highly infective ${ }^{5}$. Though harmless, certain strains of coliforms could threaten humans and wildlife, and the bacterium is found in the gastrointestinal tracts and feces of warm-blooded animals. This is released into the environment through fecal deposition and therebyentering the water bodies due to human activities and climatic factors. In normal conditions, the strains of E. coli are suppressed by the strong acids in the intestines during digestion. Yet, certain strains like O157:H7 are the common factors in inducing diarrhoeic E. coli, which absorb nutrients from the intestinal walls and produce needle-like structures causing toxins to cause diarrhoeic conditions ${ }^{6}$.

There are various methods to detect bacterial concentration in food, milk, and water samples. The biochemical tests in the detection of bacteria, Pour Plate Method and Culturing Method are the prevalent measurement techniques used ${ }^{7}$. The enzyme and non-enzyme linked immunoassays, polymerize chain reaction (PCR) tests, voltammetric and amperometric methods, and optical detection tests like spectroscopy and fluoroscopy were

\footnotetext{
${ }^{1}$ Department of Biomedical Engineering, Karunya Institute of Technology and Sciences, Coimbatore, Tamil Nadu 641 114, India. ${ }^{2}$ Department of Robotics Engineering, Karunya Institute of Technology and Sciences, Coimbatore, Tamil Nadu 641 114, India. ${ }^{3}$ Department of Millets, Tamil Nadu Agricultural University, Coimbatore, Tamil Nadu 641003, India. 'Department of Applied Chemistry, Karunya Institute of Technology and Sciences, Coimbatore, Tamil Nadu 641 114, India. ${ }^{凶}$ email: jebasinghb@karunya.edu
} 
also used in later stages. However, in all these determination methods the factors which affect detection varied with the performance criteria for a specific test. The various factors that affect the efficiency of detection are bacterial concentration, reacting agents, antigen-antibody bond formation, the relative amount of sample usage, and detection time ${ }^{8}$. The time for detection of bacterial contamination in water is a major challenge in assuring the water quality of any region. To address the issue, the development of a $2 \mathrm{D}$-nanomaterial-based sensor is an ideal choice for the rapid, precise, live detection of pathogen contaminants (in colony formation unit) in water and calibrates the detection limits to assure accuracy with the science and technology tool.

In this view,Graphene, an allotrope of carbon with two-dimensional hexagonal lattices could be used as a nanomaterial for the proposed applications as it is $2 \mathrm{D}$-layered with a large surface area ${ }^{9}$. Recent researchers have enlightened the biocidal properties of graphene in the detection and destruction of harmful bacteria ${ }^{10}$. To overcome the limited usage of graphene, the functionalized graphene oxides are found to be an effective nanomaterial for grafting various pathogenic antigens. The rigorous process of oxidation, exfoliation, and vapor deposition alters the physical, electrochemical, mechanical, and thermal properties of these molecular compounds. Also, the amine-functionalized graphene oxide based on 2D nanomaterials possessed a high degree of interlayer $d$-spacing with a change in the stacking of GO structures ${ }^{11}$. The ease of synthesis and less toxic properties make the polydimethylsiloxane (PDMS) remarkably used for many commercial portable sensor fabricationsdue to its nature of optically clear, inert, non-toxic, silicone-based biocompatible, and recyclable polymer ${ }^{12}$. Morteza et. al. ${ }^{13}$ have established quality assurance and quality control procedures of the biosensors for near real-time monitoring of E.coli in drinking water. Govindasamy et.al. ${ }^{14}$ have reported the $\mathrm{GO}_{-} \mathrm{SrWO}_{4}$ nanocrystals incorporated $\mathrm{GO}$ sheets for the arsenic drug detections in food products. The screen printed $\mathrm{GO}-\mathrm{Mn}_{3} \mathrm{O}_{4}$ microcubes based nanocomposite was developed as a modified electrode for the detection of low-level nitrates ${ }^{15}$. Wu et. al. ${ }^{16}$ have innovated the rapid enzyme-based detection technique of $E$. coli using a membrane-based approach. Chromogenic substrates were used to optimize the enzyme hydrolysis and its sample concentrations. The detection of $E$. coli from milk samples and in-situ monitoring involves the use of a microelectrode array without an $E$. coli growth medium was also demonstrated ${ }^{17}$. The monitoring of the bacterial concentration at the water treatment site was also established in lakes in Norway, the equipment itself has to be incorporated with the plant, for regular monitoring levels ${ }^{18}$. Wang et.al. ${ }^{19}$ have demonstrated the modified electrode of GO decorated ZnO nano-flowers for the quantification of 8-HDG in human urine samples. Besides, all the existing methods are offline, involves the collection and carrying of test samples to the laboratory where it is tested. Many detection techniques have been focused on the measurement of optical ${ }^{20}$, electrochemical ${ }^{21}$, andpiezoelectrics methods ${ }^{22}$. The embedded use of nanotechnology in wireless sensor networks results in theinnovative automated strategies in water quality analysis ${ }^{23}$. Mani et al. ${ }^{24}$ have reported the real-time analysis of the electrochemical sensor fabricated from GO$\mathrm{Co}_{3} \mathrm{O}_{4}$ polyhedron nanocomposites for the detection of $\mathrm{H}_{2} \mathrm{O}_{2}$ assay with the detection limit of 15 nM.Encapsulation of $\mathrm{CuFeS}_{2}$ in the GO planes was developed for the detection of methyl parazoxone (MOX) in the vegetables. The non-enzymatic sensor was used for the real time analysis of the detection of MOX with the detection limit of $4.5 \mathrm{nM}^{25}$. Herein we report the functionalized GO material-based nanosensor for the low-level detection of E. coli concentration in freshwater sources. The AD5933 impedance analyzer was used to standardize, with the frequency range of $0.01 \mathrm{~Hz}$ to $100 \mathrm{kHz}$. The obtained analog data from the functionalized GO-based nanosensor was programmed with a microcontroller and the bacterial concentration in colony-forming units (CFU) was displayed for real-time analysis. Literature manifests the formidable situation of the lakes alongside the Noyyal River across the Coimbatore district in Tamil Nadu, India. Instead of this, less than 10 lakes in the above region were considered for the contamination analysis ${ }^{26}$.

\section{Results and discussion}

The electrical conductivity of carbon and its derivatives had a great impact on the development of nanosensors and nanomaterials. The enhanced interlayer $d$-spacing distance and the chemical properties of $s p^{2}$-hybridized carbon make it even more useful for device fabrication ${ }^{27}$. The challenges of the expensive and tedious manufacturing process of monolayer graphene restrict its usage in many industrial applications. To prevail over these negative features, graphene oxide is used widely, which is easier and cheaper to manufacture than graphene as such.

It also helps in enhancing physical and chemical properties like tensile strength, elasticity, conductivity, and so on. GO comprises three functional groups such as epoxide, alcohols, and terminal acids. The oxygenated functional groups of GO can be further modified to manipulate the properties of the graphene sheets. The amine-functionalized GO material was used to develop the sensor due to the enhancement of its $d$-interlayer spacing as shown in Fig. 1. The intercalated GO was identified for the sensing applications due to the enhanced heteroatoms on the surface which creates a significant room of coordination. The $f G O$ material displays enhanced wettability and hydrophilic in nature, which eventually interacts the biological molecules with high polarity. Therefore, the efforts have been made to intercalatethe protected Diethylenetriamine (DETA) and subsequent deprotection strategy facilitates the covalent attachment of DETA, which contain heteroatoms such as nitrogen, and oxygen on the basal planes of GO. The unique morphology and textural properties are the key advantages of the covalently grafted GO. Particularly, grafting organic molecules on GO could lead to additional surface modification, resulting in the change of electronic properties.

Furthermore, covalent approaches offer the excessive benefit of attaining long-lasting stabilization of the isolated graphene sheets. The functionalization could improve the properties of GO such as surface charge, wettability and electron mobility, and hence conductivity. The developed intercalated GO nanosheets are used as the electrode material for the detection of pathological bacterial contaminants in water. On the other hand, E. coli is the most commonly found bacterial species, which produces Shiga toxins and it is gram-negative. 

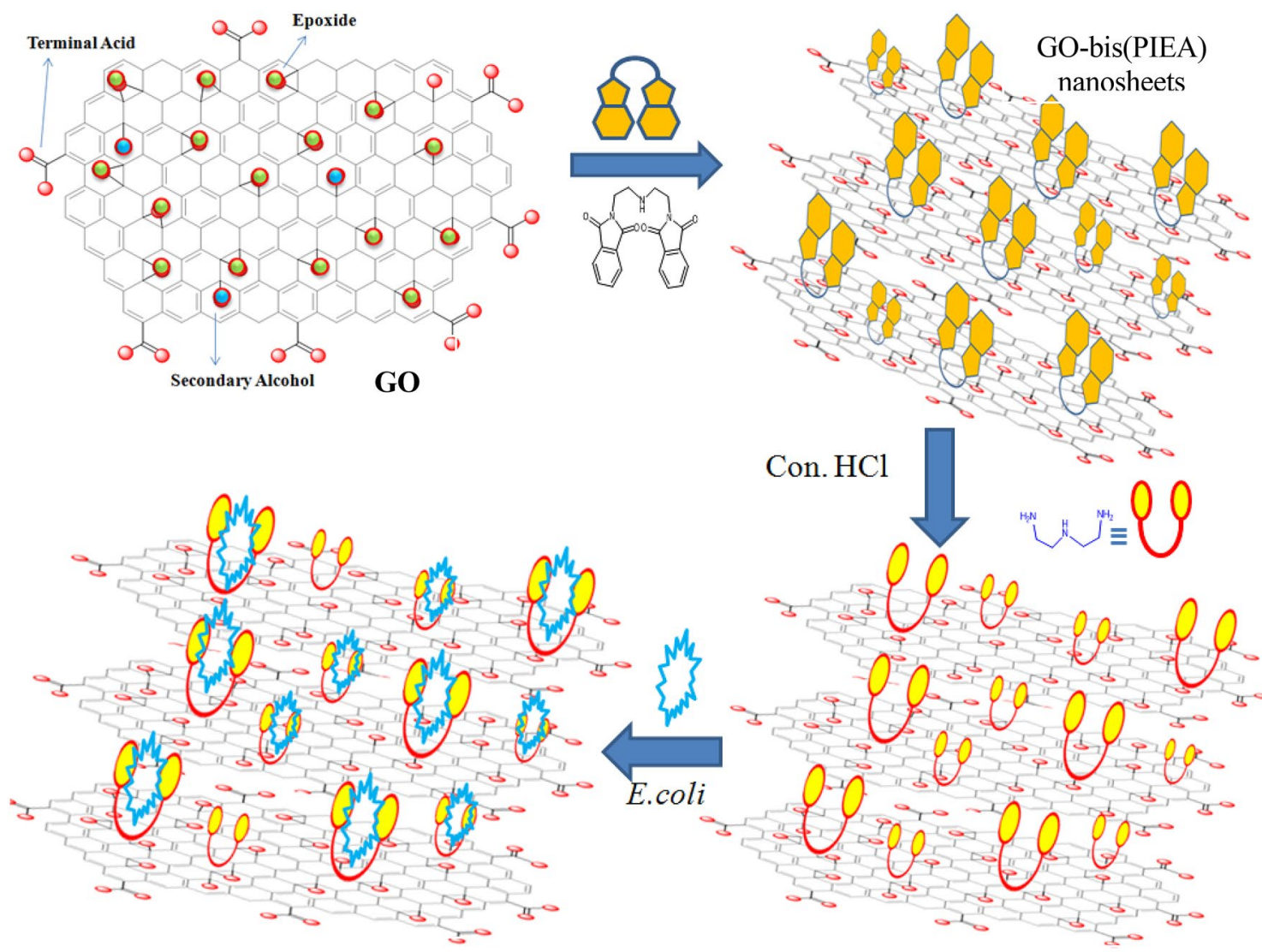

E.coli detected $f \mathrm{GO}$ nanosheets

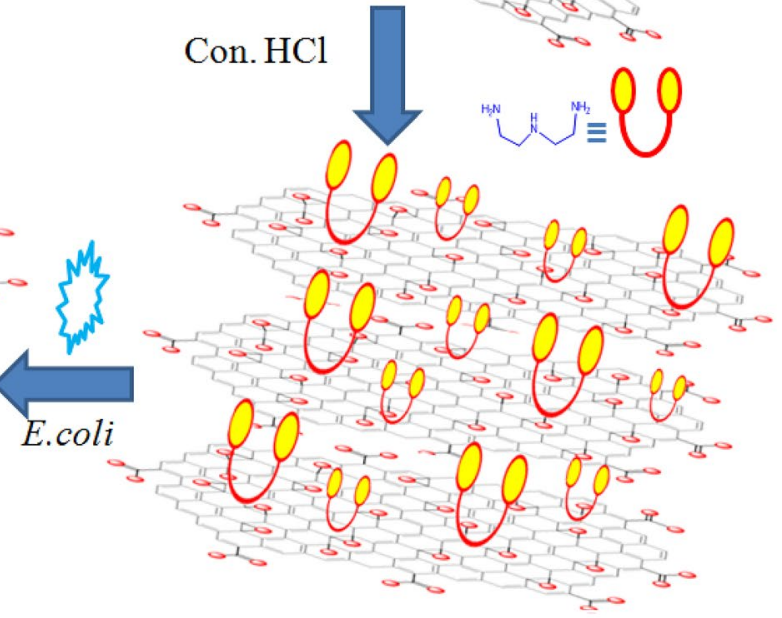

GO-DETA Nanosheets

Figure 1. Schematic illustrations of amine functionalized GO for sensing application.
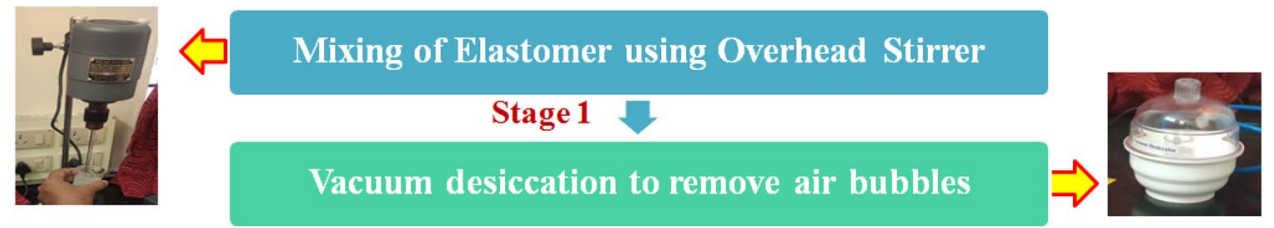

$$
\text { Stage } 2
$$

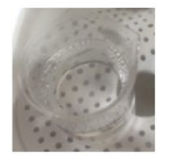

\section{$\longleftarrow \quad$ Loading solution after desiccation}

Stage 3

\section{Preparation of microfluidic structure}

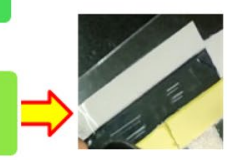

Stage 4
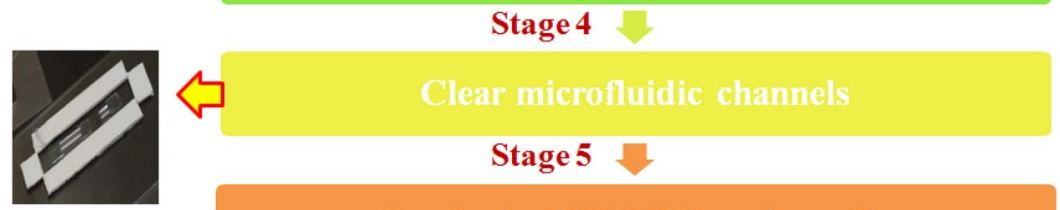

\section{Synthesized PDMS based test kit}

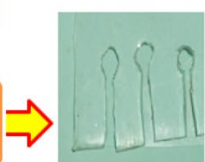

Figure 2. Process of development of PDMS-based test kit for the detection of $E$. coli in water quality analysis.

It causes illness and bacterial disorders, which are most detrimental to infants. The Heart-Brain Infusion (HBI) Broth was taken to culture E. coli samples (see Supplementary Fig. S10a-c). The stepwise device fabrication process of the nanosensor was displayed in Fig. 2.

The polymeric PDMS substrate was fabricated by blending with the curing agent using an overhead stirrer. The residue was desiccated overnight under a vacuum to remove the air bubbles. The resulting transparent loading 

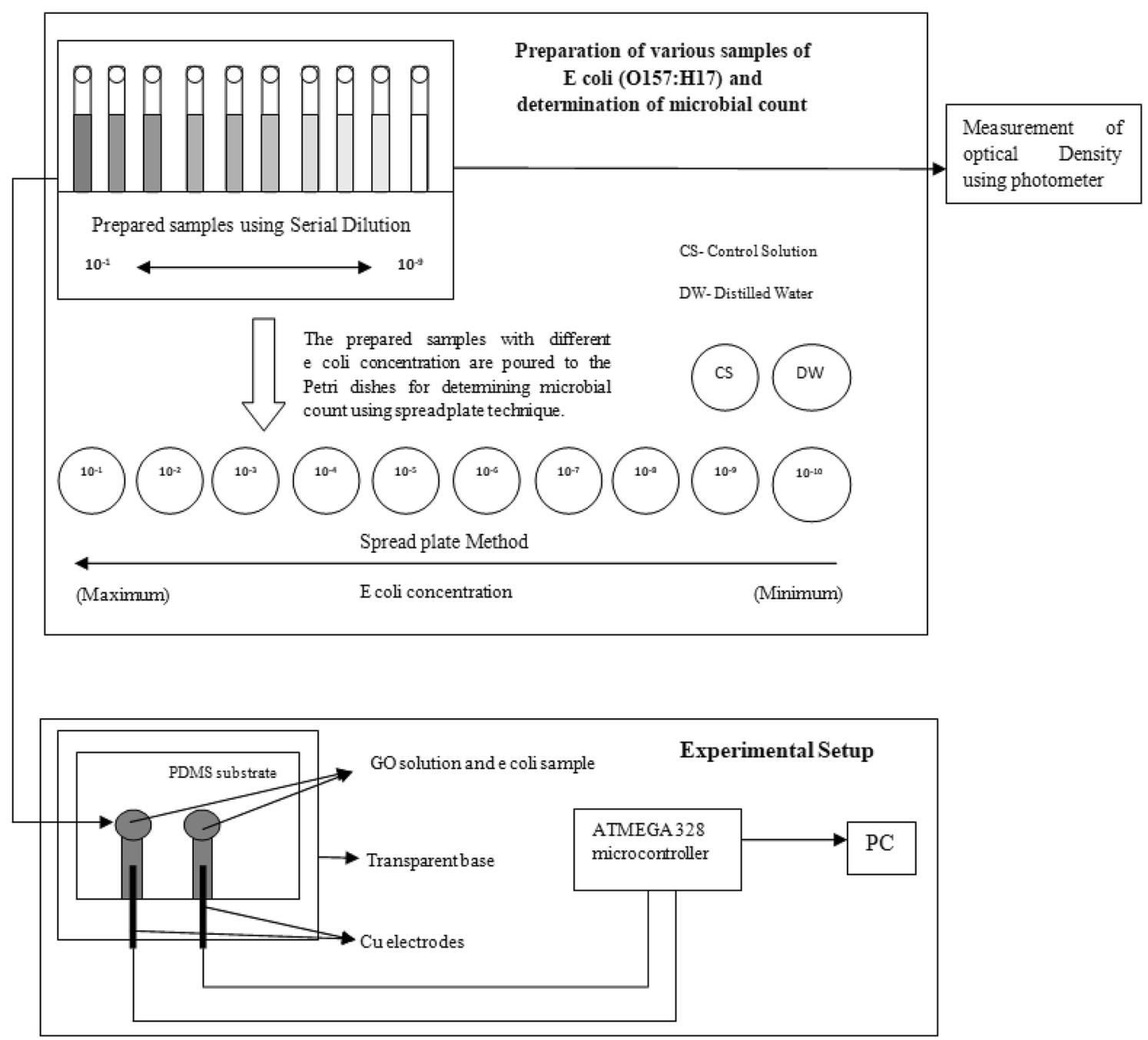

Figure 3. Outline of synthesis of bacteria, determination of microbial count, and nanosensor-based experimental setup.

fluid was transferred into the microfluidic template module and allowed to cure to get PDMS based test kit. The as-developed test kit was channelized with the functionalized GO and the lead for the sensing experiments. The experiment was performedoffline, using the developed nanosensor to measure the bacterial count/CFU. The procedure of the experiments underwent was illustrated in Fig. 3. The copper electrodes were connected to the analog pin of the microcontroller which reads the analog voltage. The inbuilt analog to digital converter in the controller produces step changes for each analog voltage value, which in turn was an indication of the concentration change.

The bacterial count was determined and the optical density was also measured. The real-time implementation of the work could be done only after proper offline studies and analysis. The experiment setup consists of the PDMS-based nanosensor; Copper wire (1 mm thickness) was used as working electrode, data acquisition using ATMEGA 328 development board and computer for interpretation and analysis. Since the majority of the samples were contaminated water samples, it was necessary to study the hydrophobic nature of the sensor developed ${ }^{28}$. If the sensor was found to be hydrophobic the tendency to clog between the surfaces prevails, which makes the sensor less effective. The hydrophobic test was analyzed and was shown in Fig. 4a. The voltage measurement using a microcontroller was depicted in Fig. $4 \mathrm{~b}$. The figure also shows the distilled water sample, E. coli sample, and GO-based nanosensor solution in glass tubes. Figure 4c,d represent the GO before and after mixing with E. coli samples. The efficiency of the sensor was tested using the real water sample from the lakes in the region of interest, which eliminate the process of serial dilution. The collected samples were analyzed for the determination of bacterial count using the spread plate technique, and the impedance measurements were performed in parallel to the contamination count.

The chemical reactions of the bacteria with the nanomaterial were analysed ${ }^{29}$. It was observed that the dilution of the bacterial solution with $f G O$ solution has resulted in an interlocking through van der Waals interactions. The change in voltage was due to the change in the conductivity of the developed $f G O$ nanosheets when interacted with $E$. coli. This is in fact due to the change in impedance offered by the $f G O$ nanosheets. An efficient method to measure impedance is necessary at this juncture. A simple cost-effective impedance analyzer made 
(a)

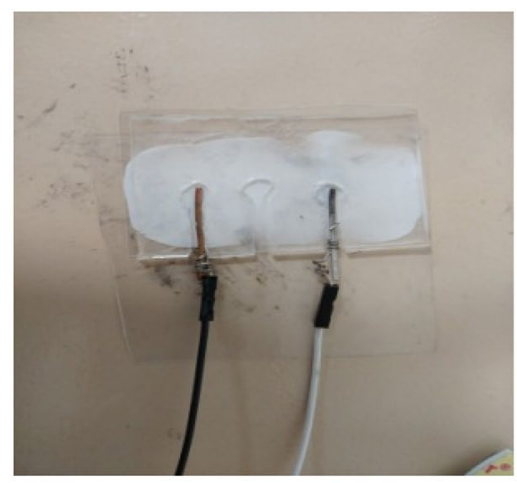

(c)

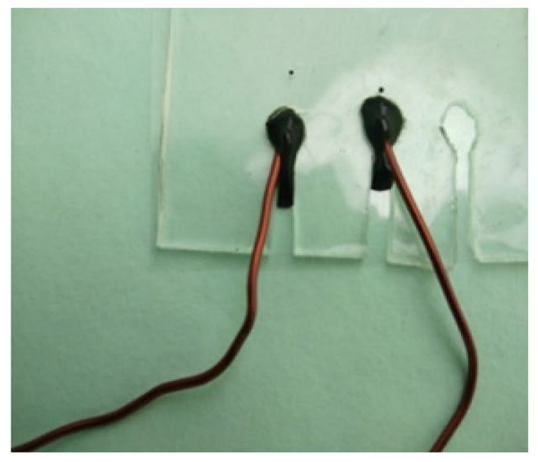

(b)

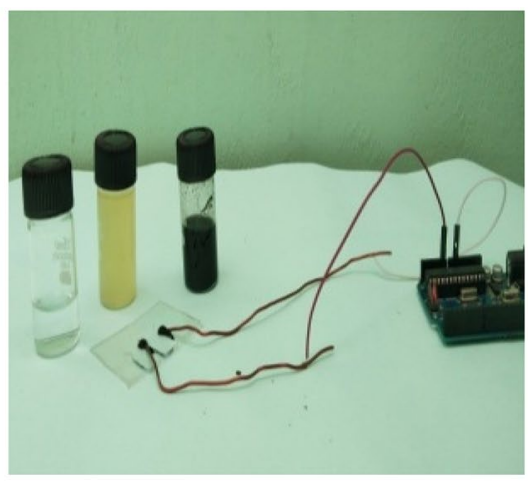

(d)

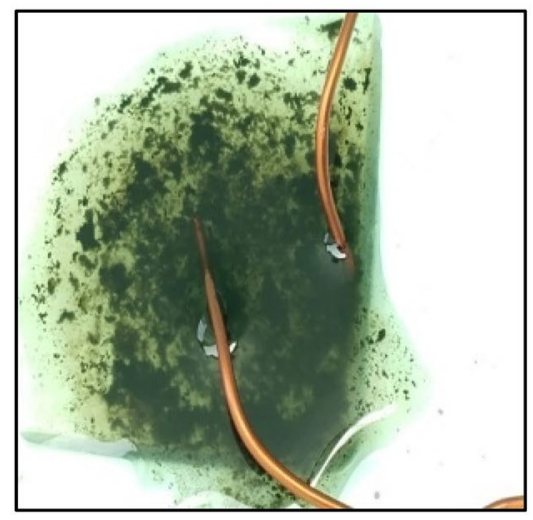

Figure 4. Experimental setup comprising of the following: (a) Hydrophobic testing of the sensor base; (b) PC-based analysis of normal water samples; (c) Voltage measurements with Cu electrodes; (d) E. coli samples inoculated in $f G O$ solution.

of AD5933 was used. The low power consumption and other exemplary features of AD5933, when compared to standard impedance spectroscopic measurement, make it more reliable to use for data monitoring. The inbuilt frequency generator is used to excite frequencies through the electrodes. The output current signal was fed to a trans-impedance amplifier to read the output in voltage. These voltage values were read by the processor and were sampled at each frequency of excitation. The stored sample data was analyzed and sent to the microprocessor through the $\mathrm{I} 2 \mathrm{C}$ interface ${ }^{30}$. It was observed that the impedance was unknown for few frequency ranges, though the output currents were produced.

The limitations of the above method, the analyzer circuit were modified by adding a high impedance application-based amplifier, viz, AD8646. This change facilitates the reductionof the cycling effects and also enhances the frequency range of measurement. Two impedance analyzer chips were used to measure the voltage across the unknown impedance and the current. To aid the AD8646 amplifiers were used. Depending on the frequency produced by the impedance analyzer, voltage is produced. The magnitude coefficients of the output were sent to the second analyzer, which measures these magnitude components proportional to the current. An external clock pulse was also used to synchronize data acquisition between two impedance analyzers.

The experimental results were outlined in Table 1 . In the initial stages of the experiment, different bacterial samples were taken from the $E$. coli cultured nutrient agar medium ${ }^{31}$. The amine-functionalized GO nanosheets dispersed mixture was kept at a constant value $(0.6 \mathrm{~mL})$. For easy analysis, $0.2 \mathrm{~mL}$ of bacteria sample was inoculated to $f \mathrm{GO}$ solution and the procedure continued till a significant change in voltage was visualized. It was observed that as the bacterial concentration increases, the step value also increases, increasing voltage.

This interprets that, the $E$. coli antigens were intercalated in the interlayer spacing of $f G O$ nanosheets and as it gets locked, the somatic characteristics of the coliforms change and it gets bound towards the $f \mathrm{GO}$ nanosheets as an interlocking system, in turn changing the electrical conductivity of the graphene sheets. The impedance of the sensor increases, which results in a high potential drop across the bonds and thus an increase in voltage. The initial readings were taken from distilled water and normal tap water. The polarity of distilled water ranges from -0.10 to -0.11 and tap water was found to be at -0.12 to -0.13 . The readings for the GO solution were observed from -0.14 to -0.15 . All these readingswere in $\mu \mathrm{V}$.

The experiments were also performed to analyze the sensor voltage at different ratios of $f \mathrm{GO}$ nanosheets dispersed solution and E.coli solution. Table 2 represents the various analyze done for different weight equivalent ratios of $f \mathrm{GO}$ dispersed solution to $E$. coli sample. The measurement was restricted to less sample content (less than $4 \mathrm{~mL}$ ) due to the limitations in the determination of the optical density of the sample under test. The bacterial concentrations from the plate method and deriving from optical density were identical. A correlation 


\begin{tabular}{|c|c|c|c|}
\hline \multicolumn{2}{|c|}{ The concentration of the solution } & \multirow[b]{2}{*}{ Step value obtained from ADC of Arduino microcontroller } & \multirow[b]{2}{*}{ Voltage acquired in multimeter $(\mu \mathrm{V})$} \\
\hline Sample & $\begin{array}{l}\text { Bacterial concentration }(0.6 \mathrm{~mL} \text { of } f \mathrm{GO} \text { solution added to } \\
\text { E. coli solution) ascending (in mL) }\end{array}$ & & \\
\hline CONC 1 & 0.2 & 244 & -0.17 \\
\hline CONC 2 & 0.4 & 256 & -0.13 \\
\hline CONC 3 & 0.6 & 253 & -0.10 \\
\hline CONC 4 & 0.9 & 261 & +0.01 \\
\hline CONC 5 & 1.2 & 261 & +0.07 \\
\hline CONC 6 & 1.5 & 264 & +0.11 \\
\hline CONC 7 & 1.7 & 264 & +0.17 \\
\hline CONC 8 & 1.9 & 264 & +0.23 \\
\hline CONC 9 & 2.1 & 267 & +0.38 \\
\hline CONC 10 & 2.3 & 273 & +0.44 \\
\hline
\end{tabular}

Table 1. Experimental data for various bacterial concentrations.

\begin{tabular}{|c|c|c|c|c|}
\hline Distilled water (in $\mathrm{mL}$ ) & E. coli (in mL) & Optical density & Step change in microcontroller & Voltage output (mV) \\
\hline \multicolumn{5}{|c|}{ Amine functionalized $f \mathrm{GO}$ based nanosensor solution, $0.05 \mathrm{~mL}$} \\
\hline 1.5 & 1.5 & 1.29 & 269 & -0.19 \\
\hline 2 & 1 & 0.9 & 267 & -0.19 \\
\hline 2.5 & 0.5 & 0.86 & 267 & -0.18 \\
\hline 2.8 & 0.2 & 0.8 & 266 & -0.17 \\
\hline 3 & 0 & 0 & 265 & -0.17 \\
\hline \multicolumn{5}{|c|}{ Amine functionalized GO-based nanosensor solution, $0.1 \mathrm{~mL}$} \\
\hline 1.5 & 1.5 & 0.79 & 265 & -0.17 \\
\hline 2 & 1 & 0.65 & 265 & -0.16 \\
\hline 2.5 & 0.5 & 0.51 & 264 & -0.16 \\
\hline 2.8 & 0.2 & 0.45 & 262 & -0.15 \\
\hline 3 & 0 & 0 & 260 & -0.15 \\
\hline \multicolumn{5}{|c|}{ Amine functionalized GO-based nanosensor solution, $0.5 \mathrm{~mL}$} \\
\hline 1.5 & 1.5 & 0.41 & 259 & -0.14 \\
\hline 2 & 1 & 0.37 & 257 & -0.14 \\
\hline 2.5 & 0.5 & 0.28 & 256 & -0.13 \\
\hline 2.8 & 0.2 & 0.15 & 255 & -0.13 \\
\hline 3 & 0 & 0 & 253 & -0.12 \\
\hline
\end{tabular}

Table 2. Experimental data for varying molar weight of antigen and antibody solutions.

was made to the step change (Voltage data was not steady and might result in false reading) to the bacterial concentration. The least concentration sample was counted to have a concentration of $600 \times 10^{8} \mathrm{CFU} / \mathrm{mL}$.

The use of carbon fiber electrodes was used in onsite monitoring resulting in a detection range of $10^{3}-10^{8} \mathrm{CFU} /$ $\mathrm{mL}$. Though detection time was less than $5 \mathrm{~h}$, the study focussed on the change in concentration with respect to time and the effectiveness of the sensor to detect the least and maximum time-concentration relation ${ }^{32}$. The higher the contamination, the more difficult it to estimate the count using the spread plate ${ }^{33}$. Also, it was realized that if the volume of GO solution exceeds $1 \mathrm{~mL}$ while testing $\mathrm{OD}$, the measurement was null due to the low clearness/turbidity of the water sample. From the above tables, it was identified that there was a decent variation in impedance with the changes in bacterial contamination and the reagent substrate, say, GO solution. A desirable change in voltage is visualized in Table 1 . This is due to the high molar fractions of $E$. coli and fGO.Though the voltage changes are negligible, the change in step value derived from ADC shows a significant variation in impedance as observed in Tables 2 and 3.

However, the primary objective of the study was to develop a sensor for the analysisof the contamination extremities in a complex water matrix. Though the prepared water samples were gathered from the foothills of the Siruvani River, there were other predominant contaminated water sources within the same geographical area. Tamil Nadu Water Supply and Drainage Board (TWAD) has identified about 449 among 2476 were contaminated sources in the region of the study performed inthe Coimbatore district. A major portion of the water bodies constitutes the wetlands and lakes which reserve water from basins of the Noyyal riveralongside the district. These lakes have a major role in maintaining the ecosystem of the region. Literature depicts that majority of these lakes were the primary source of water for the urban populated cities and are highly polluted. Hence continuous 


\begin{tabular}{|c|c|c|c|}
\hline \multicolumn{2}{|l|}{ E. coli sample $1 \mathrm{~mL}$, distilled water $2 \mathrm{~mL}$} & \multirow[b]{2}{*}{$\begin{array}{l}\text { Step value obtained from ADC of Arduino } \\
\text { microcontroller }\end{array}$} & \multirow[b]{2}{*}{ Voltage acquired in multimeter $(\mu \mathrm{V}$} \\
\hline $\begin{array}{l}\text { Amine functionalized GO-based nanosensor solution } \\
(\mathrm{mL})\end{array}$ & Optical density & & \\
\hline 0 & 0.06 & 269 & -0.16 \\
\hline 0.1 & 0.07 & 265 & -0.15 \\
\hline 0.2 & 0.19 & 268 & -0.15 \\
\hline 0.3 & 0.28 & 262 & -0.14 \\
\hline 0.4 & 0.47 & 260 & -0.13 \\
\hline 0.5 & 0.64 & 262 & -0.13 \\
\hline 0.6 & 0.76 & 257 & -0.11 \\
\hline 0.7 & 1.06 & 256 & -0.09 \\
\hline 0.8 & 1.34 & 255 & -0.08 \\
\hline 0.9 & 1.76 & 252 & -0.07 \\
\hline 1 & $>2$ & 251 & -0.08 \\
\hline
\end{tabular}

Table 3. Experimental data for varying molar weight of $f \mathrm{GO}$.

\begin{tabular}{|c|c|c|c|c|c|}
\hline \multirow[b]{2}{*}{ Sample number } & \multirow[b]{2}{*}{ Optical density } & \multirow[b]{2}{*}{ E. coli count using agar plate } & \multicolumn{3}{|c|}{ E. coli sample $0.5 \mathrm{~mL}$ mixed with $0.5 \mathrm{~mL}$ of functionalized GO solution } \\
\hline & & & Optical density & $\begin{array}{l}\text { Step value obtained from ADC of Arduino } \\
\text { microcontroller }\end{array}$ & Voltage acquired in multimeter $(\mu \mathrm{V})$ \\
\hline 1 & 0.46 & 140 & 1.93 & 19 & 0.0927 \\
\hline 2 & 0.51 & 182 & 1.62 & 25 & 0.1220 \\
\hline 3 & 0.34 & 187 & 1.79 & 21 & 0.1025 \\
\hline 4 & 0.47 & 182 & 1.89 & 26 & 0.1269 \\
\hline 5 & 0.53 & 130 & 1.92 & 21 & 0.1025 \\
\hline 6 & 0.62 & 111 & 1.87 & 24 & 0.1171 \\
\hline 7 & 0.53 & 123 & 1.89 & 23 & 0.1123 \\
\hline 8 & 0.47 & 194 & 1.94 & 24 & 0.1171 \\
\hline 9 & 0.39 & 183 & 1.79 & 22 & 0.1074 \\
\hline 10 & 0.51 & 173 & 1.46 & 26 & 0.1269 \\
\hline
\end{tabular}

Table 4. Experimental data for a real water sample and using developed nanosensor-laboratory analysis.

monitoring of these sources is most needed to ensure safe water consumption. 10 among 24 lakes in Coimbatore were inspected and the Geographical Information System (GIS) represents the location of the lakes identified.

An efficient mapping tool called Geographical Information System (GIS) was used to illustrate the location of lakes identified for E. coli detection (see Supplementary Fig. S14). It was noteworthy to report that all the lakes identified were either developed or naturally occurred and filled up due to the climatic and human interventions. Most of these water bodies were located within the heart and the outskirts of the city and were the primary source of water for the urban population and industries. Hence, there arises a need for quality monitoring of these water sources. Conventional quality testing comprises of testing the basic water parameters like $\mathrm{pH}$, turbidity, and Total Dissolved Solvents which were comparatively easy to detect. As stated above, E. coli detection was time-consuming and the effect of contamination affects the victims more rapidly. Hence, an effective system also facilitates to address the problems associated with water source which was commonly utilized by the inhabitants.

The water samples were analyzed at the lake site for impedance change and voltage detection. The same was carried to the laboratory to analyze the bacterial count and to calibrate with the colorimeter to check the optical density. The procedure and methods were similar to the experiments conducted for developed E. coli samples using known strains (see Supplementary Fig. S17). As illustrated in Tables 1, 2 and 3, known E. coli samples with varying concentration was used to study the effectiveness of the sensor. The efficiency of the sensor was also tested using the real water samples and the results were depicted in Table 4 . As the samples were collected from a different geographical region, the orderly severity of contamination cannot be shown. However, the laboratory investigations prove that each of the identified lakes was contaminated in various levels in the range of less than $200 \times 10^{2} \mathrm{CFU} / \mathrm{mL}$.

On analysis, it may be interpreted that the known E. coli samples possess an impedance variation in accordance with the concentration of the bacteria. Whereas, the real sample collected from the water bodies also exhibits a change in impedance as clearly depicted in the table, a change in voltage and optical density does exist. This can be even more proved by understanding Table 4 . The idea of using functionalized GO was to link the bonding of $E$. coli bacteria, hence no other bacterial source or contaminants can give way to the rise of potential or density or a mere change in impedance. Hence the method can be effectively used in E. coli detection in less time. However, the application of artificial intelligencein the future can even make the sensor more efficient so 
that the E. coli count and impedance change can be more interrelated using a trained set of data through an appropriate learning strategy.

\section{Conclusions}

In this work, a unique class of intercalated graphene-based nanosensors was developed, to detect the E. coli at very low concentrations in the complex water matrices. For the experimental studies, the bacterial count (CFU) of synthesized E. coli samples and real water samples were counted and analyzed. The temperature variations and other unpredictable factors may affect the physical and chemical properties of the bacteria under test. Hence, there was a need of developing a technique to measure the coliforms content in the real-time analysis platform to access the impact on the aquatic environment. The device fabrication was executed with the PDMS polymeric substrate with microfluidic channels for the electrodes. When the sensor contacts the aquatic environment along with the increase of $E$. coli bacterial contamination, the output voltage was also increased. This led to the development of rapid detection of $E$. coli bacteria using nanosensors by the fabrication of amine-functionalized GO nanosheets. These nanosheets facilitate the larger interlayer $d$-spacing for the selective grafting of $E$. coli in low concentration detection. Among the sensors available in the market, the majority are expensive and bulky. To overcome these limitations, a nanosensor was developed with high efficiency and rapid detection of bacterial concentration. Though the development of one such sensor is costly, mass production utilizing $3 \mathrm{D}$ printed polymers helps in commercializing the product and its usefulness to a great extent.

\section{Experimental}

Materials and methods. The samples of known concentrations of E. coli contaminated water were prepared using the serial dilution method. The microbial count of these samples is determined using Spread Plate Method. These samples were inoculated to the functionalized GO nanomaterial in various molar ratios, and the voltage values were noted. For ensuring better calibration, the optical densities of these samples were also measured simultaneously. The water samples from various water matrices were collected and tested using the developed sensor for real water analysis. Conc. $\mathrm{H}_{2} \mathrm{SO}_{4}$, sodium meta periodate, ethanol, tetrahydrofuran, $\mathrm{H}_{2} \mathrm{O}_{2}(30 \%)$, Diethylenetriamine (DETA), and phthalic anhydride were purchased from Sigma-Aldrich. Polydimethylsiloxane (PDMS) is a silicone-based organic polymer used for the fabrication of nanosensors ${ }^{34}$. The silicone elastomer curing agent and its elastomer base were purchased from Sigma Aldrich. The protected DETA functionalized $\mathrm{GO}$ and free amine-functionalized $\mathrm{GO}(f \mathrm{GO})$ were prepared by the previously reported procedure ${ }^{35}$.

Preparation of bacteria culture. The strains of E. coli Shiga toxins were cultured in a liquid broth medium and inoculated to develop in higher concentration ${ }^{31}$. The Heart-Brain Infusion (HBI) Broth was taken to culture E. coli samples. The standard laboratory preparation method ${ }^{36}$ was followed to culture the bacteria in the general-purpose HBI medium. The constituents of the medium were rich in carbon, nitrogen, and essential amino acids and vitamins enhancing the growth. In the media, dextrose, sodium chloride, and disodium phosphate act as the source of energy, equilibrium balancing, and buffering agents, respectively.

The procedure for the preparation of the broth was as follows: suspend $37.0 \mathrm{~g}$ of nutrient medium powder in $1 \mathrm{~L}$ of deionized water. Stir the solution until the medium dissolves completely. After dispensing the required quantity, the mixture was placed in an autoclave at $121^{\circ} \mathrm{C}$ for $15 \mathrm{~min}$. The prepared culture medium is stored at a very low temperature in a sealed container. This is the procedure of growing E. coli in the culture medium (BHI broth): The prepared medium was allowed to acclimatize to the room temperature, further it was inoculated using O157:H17 strains. The medium was incubated in closed containers for preventing external contaminants and the growth was examined after $48 \mathrm{~h}$. After the incubation period tubes were examined for turbidity which was an indication of the growth. Later, the culture broth was sub-cultured to various test samples in different concentrations and the colonies were observed (Fig. 2) by Pour Plate Method.

Preparation of bacterial samples by serial dilution method. The experiment was performed with ten samples with varying $E$. coli concentrations and the sample solutions were prepared using serial dilution which involves the process of mixing the known amount of sample to deionized water resulting in one concentration ${ }^{37}$. $1 \mathrm{~mL}$ of prepared E. coli medium was inoculated to $9 \mathrm{~mL}$ of distilled water, resulting in the sample with the highest concentration. $1 \mathrm{~mL}$ from the new sample mixture was inoculated to $9 \mathrm{~mL}$ of distilled water to give $1 / 10$ th molar concentration of the parent sample. The process was repeated several times to obtain the number of samples with various concentrations. The dilution factor of $1: 10$ or $10^{-1}$ was added after each dilution, and thereby reducing the concentration. The known volumes of these samples were spread to the nutrient medium, and by identifying the number of colonies formed, the microbiological count was determined.

Spread plate method. The sub-cultured bacteria samples were poured into the Petri dishes for the bacterial count ${ }^{38}$. The Petri dishes that contain E. coli samples at various concentrations were incubated at a warm temperature between $\left(35-37^{\circ} \mathrm{C}\right)$ for $48 \mathrm{~h}$ or more, but not more than $70 \mathrm{~h}$ (more time-lapse will enhance the bacterial growth in Petri dish). The number of visible bacterial colonies was counted either using markers or using manual counting for a square area using a scale marker. The number of the viable bacterial cell was measured as colony formation unit (CFU) was expressed as.

$$
\text { Bacteria }(\mathrm{CFU}) / \mathrm{mL}=\text { (no. of colonies } \times \text { dilution factor }) / \text { volume of culture plate. }
$$

Thereby, the bacterial concentration in CFU was estimated. The collected water samples were also analyzed using the standardized technique. 
Development of PDMS substrate. The process was executed with the blending of PDMS, elastomer base, and elastomer curing agent (thickener) in a ratio of 10:1. The proper mixing was ensured by using a head stirrer with a nominal speed of about $100 \mathrm{rpm}$ until the thickener and base material dissolves perfectly. After the dilution of the curing agent to the base elastomer, a thick solution full of foam was obtained which should be desiccated for removing the air bubbles. A vacuum desiccator was used at normal pressure of about 500$600 \mathrm{mmHg}$. After few minutes the vacuum inside the desiccator was liberated which also helps in popping the bubbles from the mixture. The procedure is repeated until to get a clear solution.

A $10 \mathrm{~cm} \times 4 \mathrm{~cm}$ glass slide was used as the base for the PDMS substrate. The hydrophobic nature of the PDMS base tends to repel the water containing the bacterial content. Hence to hold the sensing material and the sample, microfluidic channels have to be imparted before pouring onto the glass plate similar to most of the wearable nanosensors and biomaterials that were developed ${ }^{39}$. The general purpose adhesive tape was mounted on the sides of the glass plate to restrict the flow of the solution within the glass boundary. Three thin glass strips ( $1 \mathrm{~mm})$ were kept horizontally to the glass slide for the microfluidic space as illustrated in Fig. 3 (Stage 3). The desiccated solution was poured into the glass slide. The bubbles were removed completely and the resulting mixture is left to sit overnight to solidify. The polymer was then baked in an oven at $70-90^{\circ} \mathrm{C}$ for $1 \mathrm{~h}$. The polymer was allowed to $\mathrm{cool}$ at room temperature for a couple of hours after baking using a knife and a ruler. The edges of the adhesive tape were cut and the PDMS was peeled off. The synthesized PDMS substrate was attached to a self-adhesive polymer or plastic sheet so that the top layer shows the PDMS microfluidic channel and the bottom layer would be the polymer to support the sensor material.

Synthesis of amine-functionalized graphene oxide-based 2D nanomaterial. Synthesis of graphene oxide. Graphene Oxide was prepared by the reported method ${ }^{40}$ from graphite powder. In a typical reaction, to the stirred solution of $1 \mathrm{~g}$ of graphite powder in $40 \mathrm{~mL}$ of Conc. $\mathrm{H}_{2} \mathrm{SO}_{4}(98 \%), 12 \mathrm{~g}$ of sodium meta periodate was added at ice-cold temperature with vigorous stirring for $24 \mathrm{~h}$. The thick, grey color paste was then diluted slowly by the addition of $150 \mathrm{~mL}$ of distilled water with continuous stirring to get the color change from grey to dark brown color. To this mixture, $5 \mathrm{~mL}$ of $\mathrm{H}_{2} \mathrm{O}_{2}(30 \%)$ was added and stirred for $1 \mathrm{~h}$ with the addition of Conc. $\mathrm{HCl}(5 \mathrm{~mL})$. The brown dispersed solution was centrifuged and the brown solid GO was washed with 1:1 $(v / v)$ aqueous ethanol $(3 \times 20 \mathrm{~mL})$ to remove the water and diethyl ether $(20 \mathrm{~mL})$ to get the free flow solid. The dark brown product was dried and stored in the desiccator.

Preparation of amine-functionalized GO-DETA nanosheets. The DETA functionalized GO was prepared via three simple steps. Step 1 Phthalic anhydride $(5.7 \mathrm{~g}, 0.038 \mathrm{M})$ was heated to $120^{\circ} \mathrm{C}$ for 30 min with constant stirring, and Diethylenetriamine $(2.1 \mathrm{~mL}, 0.019 \mathrm{M})$ was slowly added to the molten state. The dense white fumes were condensed into the reaction flask. To this residue, ethanol $(100 \mathrm{~mL})$ was added and stirred for $2 \mathrm{~h}$. Upon cooling, the bis(phthalimidoethyl)-amine was formed as pale a yellow solid. It was used for the further process without purification. Yield: $94 \%$ (6.8 g). Step 2: The protected amine, bis(phthalimidoethyl)-amine was used to intercalate the GO basal planes. In a typical reaction, the well-dispersed GO (500 $\mathrm{mg}$ ) solution in $150 \mathrm{~mL}$ of de-ionized water was sonicated for $30 \mathrm{~min}$. To this dispersed solution, Bis(PIEA) amine $(1 \mathrm{~g})$ in ethanol $(40 \mathrm{~mL})$ was added dropwise under continuous stirring for $24 \mathrm{~h}$. Finally, the mixture was centrifuged at $5000 \mathrm{rpm}$ for $10 \mathrm{~min}$ to isolate the GO/bis(PIEA). The GO/bis(PIEA) solid was washed with ethanol and diethyl ether to isolate the free-flow black solid. Step 3: The protecting group phthalic acid was removed by the acid treatment. Typically, GO-bis(PIEA) $(300 \mathrm{mg})$ was stirred with $10 \%$ of $\mathrm{HCl}$ solution under RT condition for $24 \mathrm{~h}$. The GO-DETA was isolated by centrifugation of the above mixture at $5000 \mathrm{rpm}$ for $10 \mathrm{~min}$. The resultant free amine DETA functionalized GO was washed well with hot water, ethanol, and diethyl ether to isolate the black solid. The sensor solution was made by mixing $20 \mathrm{mg}$ of amine-functionalized GO material with $10 \mathrm{~mL}$ of conductivity/HPLC water. The mixture after sonication for about $15 \mathrm{~min}$ was ready for use. The intercalated materials GO-Bis(PIEA) and GO-DETA were characterized using XRD, FT-IR, XPS, RAMAN, and TEM (refer Supplementary Figs. S2-S6) for the confirmation of functionaliztionations.

Received: 17 April 2021; Accepted: 26 July 2021

Published online: 19 August 2021

\section{References}

1. Vikesland, P. J. Nanosensors for water quality monitoring. Nat. Nanotechnol. 13, 651-660 (2018).

2. Aggarwal, P., Uppal, B., Ghosh, R., Krishna Prakash, S. \& Rajeshwari, K. Highly-resistant E. coli as a common cause of paediatric diarrhoea in India. J. Health Popul. Nutr. 31(3), 409-412 (2013).

3. Subitha, L. \& Jayalakshmy, R. J. Diarrheal diseases among children in India: Current scenario and future perspectives. Nat. Sci. Biol. Med. 6(1), 24-28 (2015).

4. WHO. Waterborne Pathogens and Their Significance in Water Supplies 1-6 (World Health Organanization, 2012).

5. Progress on Drinking Water, Sanitation, and Hygiene. Update and SDG Baleshis (WHO, UNICEF, 2017).

6. Deisingh, A. K. \& Thompson, M. Strategies for the detection of Escherichia coli O157:H7 in foods. J. Appl. Microbiol. 96(3), 419-429 (2004).

7. James, G. C. \& Welsh, C. T. Microbiology: A Laboratory Manual 11th edn. (Pearson Publication, 2017).

8. Xu, H., Tang, J., Dai, J., Wang, C. \& Zhou, X. Ultrasensitive and rapid count of Escherichia coli using magnetic nanoparticle probe under the dark-field microscope. BMC Micro. Biol. 18, 100-106 (2018).

9. Le, T. N., Tran, T. D. \& Kim, M. I. A. A convenient colorimetric bacteria detection method utilizing chitosan-coated magnetic nanoparticles. Nanomaterials 10, 92-103 (2020). 
10. Parveen, K., Huo, P., Zhang, R. \& Liu, B. Antibacterial properties of graphene-based nanomaterials. Nanomaterials 9(5), 737-769 (2019).

11. Nguyen, B. H. \& Nguyen, V. H. Advances in graphene-based optoelectronics, plasmonics and photonics. Adv. Nat. Sci. Nanosci. Nanotechnol. 7, 1-8 (2016).

12. Bong, K. W. et al. Non-polydimethylsiloxane devices for oxygen-free flow lithography. Nat. Commun. 3, 1-10 (2012).

13. Nikou, H., Nugel, K. Y., Elzein, M., Absar, A. \& Morteza, A. A strategy to establish a quality assurance/quality control plan for the application of biosensors for the detection of E. coli in water. Biosensors 7(2), 1-11 (2017).

14. Govindasamy, M. et al. Investigation of sonochemically synthesized sphere-like metal tungstate nanocrystals decorated activated carbon sheets network and its application towards highly sensitive detection of arsenic drug in biological samples. J. Taiwan Inst. Chem. Eng. 114, 211-219 (2020).

15. Muthumariappan, A., Govindasamy, M., Chen, S.-M., Sakthivel, K. \& Veerappan, M. Screen-printed electrode modified with a composite prepared from graphene oxide nanosheets and $\mathrm{Mn}_{3} \mathrm{O}_{4}$ microcubes for ultrasensitive determination of nitrite. Microchim. Acta 184, 3625-3634 (2017).

16. Wu, J. et al. Rapid detection of Escherichia coli in water using sample concentration and optimized enzymatic hydrolysis of chromogenic substrates. Curr. Microbiol. 75(5), 827-834 (2018).

17. Zhang J, et. al. An on-site, highly specific immunosensor for Escherichia coli detection in field milk samples from mastitis-affected dairy cattle. Biosenors Bioelectro. 165, 112366-112375 (2020).

18. Bigham, T. et al. Microbial water quality: Voltammetric detection of coliforms based on riboflavin-ferrocyanide redox couples. J. Electrochem. Commun. 101, 99-103 (2019).

19. Govindasamy, M. et al. A novel electrochemical sensor for determination of DNA damage biomarker (8-hydroxy-2'deoxyguanosine) in urine using sonochemically derived graphene oxide sheets covered zinc oxide flower modified electrode. Ultrason. Sonochem. 58, 104622-104630 (2019).

20. Nurliyana, M. R. et al. The detection method of Escherichia coli in water resources: A review. J. Phys. Conf. Ser. 995, 012065-012077 (2018).

21. Rochelet, M. et al. Rapid amperometric detection of Escherichia coli in wastewater by measuring $\beta$-D glucuronidase activity with disposable carbon sensor. Anal. Chim. Acta. 892, 160-166 (2015).

22. Yang, L., Li, Y., Griffis, C. L. \& Johnson, M. G. Interdigitated microelectrode (IME) impedance sensor for the detection of viable Salmonella typhimurium. Biosens. Bioelectron. 19(10), 1139-1147 (2004).

23. Ruiz-Garcia, L., Lunadei, L., Barreiro, P. \& Robla, J. I. A review of wireless sensor technologies and applications in agriculture and food industry: State of the art and current trends. Sensors 9(6), 4728-4750 (2009).

24. Sakthivel, K., Govindasamy, M., Chen, S.-M., Muthumariappan, A. \& Mani, V. 3D graphene oxide-cobalt oxide polyhedrons for highly sensitive non-enzymatic electrochemical determination of hydrogen peroxide. Sens. Actuators. B Chem. 253, 773-783 (2017).

25. Umamaheswari, R. et al. Graphene oxide encapsulated 3D porous chalcopyrite $\left(\mathrm{CuFeS}_{2}\right)$ nanocomposite as an emerging electrocatalyst for agro-hazardous (methyl paraoxon) detection in vegetables. Compos. Part B. Eng. 160(1), 268-276 (2019).

26. Dineshkumar, S. \& Natarajan, M. Assessment of water quality status in the inland lakes of Coimbatore, Tamil Nadu, India. Indian J. Geo Mar. Sci. 49(7), 1280-1285 (2020).

27. Krishnan, S. K., Singh, E., Singh, P., Meyyappan, M. \& Nalwa, H. S. A review on graphene-based nanocomposites for electrochemical and fluorescent biosensors. RSC Adv. 9(16), 8778-8781 (2019).

28. Gökaltun, A., Kang, Y. B., Yarmush, M. L., Usta, O. B. \& Asatekin, A. Simple surface modification of poly(dimethylsiloxane) via surface segregating smart polymers for biomicrofluidics. Sci. Rep. 9(1), 1-14 (2019).

29. Liu, J. T., Settu, K., Tsai, J. Z. \& Chen, C. Impedance sensor for rapid enumeration of E. coli in milk samples. J. Electrochim. Acta 185, 89-95 (2015).

30. Rose, E. J., Pamela, D. \& Rajasekaran, K. Apple vitality detection by impedance measurement. Int. J. Adv. Res. Comput. Sci. Softw. Eng. 3(3), 144-148 (2013).

31. Deshmukh, A. M. Handbook of Media, Stains and Reagents in Microbiology (PAMA Publications, 1997).

32. Tryland, I. et al. On-line monitoring of Escherichia coli in raw water at oset drinking water treatment plant, Oslo (Norway). Int. J. Environ. Res. Public Health 12, 1788-1802 (2015).

33. Stevenson, K., McVey, A. F., Clark, I. B. N., Swain, P. S. \& Pilizota, T. General calibration of microbial growth in microplate readers. Sci. Rep. 6, 4-10 (2016).

34. Mauerhofer, L. M. et al. Methods for quantification of growth and productivity in anaerobic microbiology and biotechnology. Folia Microbiol. 64, 321-329 (2019).

35. Ramesh, P. \& Jebasingh, B. A facile synthesis of bis-(pththalimidoethyl)-amine-functionalized graphene oxide and its dual performance as a supercapacitor electrode and fluorescence sensor. Mater. Chem. Phys. 152, 46-54 (2019).

36. Alfred, B. \& Heidi, S. Benson's Microbiological Applications, Laboratory Manual in General Microbiology. Curr Protoc Cytom, 13th Ed. 106-110 (2015).

37. Yang, X. et al. Design of a wireless sensor network for long-term, in-situ monitoring of an aqueous environment. Sensors 2(11), 455-472 (2002).

38. Salih, N. M., Sahdan, M. Z., Morsin, M. \& Asmah, M. T. Fabrication and integration of PDMS-glass based microfluidic with optical absorbance measurement device for coliform bacteria detection. IFMBE Proc. 63, 75-81 (2018).

39. Zhao, X., Li, M. \& Liu, Y. Microbial assembly, interaction, functioning, activity, and diversification: A review derived from community compositional data. Microorganisms 7(10), 1-19 (2019).

40. Ramesh, P. \& Jebasingh, B. Facile and effective oxidation of graphite using sodium metaperiodate. Mater. Lett. 193, 305-308 (2017).

\section{Acknowledgements}

The authors would like to thank the funding supports from UGC-DAE-Consortium for Research, Kalpakkam Node (Project No: CSR-KN/CSR-98/2019-20/473), and DST-SERB (No. SB/FT/CS-169-2012). The authors thank the research scholars in Electronics and Communication Engineering,Biomedical Engineering and Applied Chemistry, KITS for their support for the development of the prototype. The authors would express their gratitude to the Technical Staff in the Microbiology Lab of the Department of Biotechnology, KITS for rendering the aid in biochemical analysis.

\section{Author contributions}

Conceptualization, materials development-G.S., L.P. and B.J.; Methodology-L.R., A.M., G.S. and B.J.; Software-A.M., I.J.; Validation, L.R., A.M., and B.J.; Formal analysis-L.R., I.J.; Investigation-L.R., G.S., L.P.; Resources-B.J.; Data curation-A.M.; Writing-original draft preparation, L.R., A.M.; Writing-review and editing, B.J.; Visualization and supervision, L.R.; Project administration, B.J.; All authors have read, reviewed and agreed to the published version of the manuscript. 


\section{Competing interests}

The authors declare no competing interests.

\section{Additional information}

Supplementary Information The online version contains supplementary material available at https://doi.org/ 10.1038/s41598-021-96539-6.

Correspondence and requests for materials should be addressed to J.B.

Reprints and permissions information is available at www.nature.com/reprints.

Publisher's note Springer Nature remains neutral with regard to jurisdictional claims in published maps and institutional affiliations.

(c) (1) Open Access This article is licensed under a Creative Commons Attribution 4.0 International License, which permits use, sharing, adaptation, distribution and reproduction in any medium or format, as long as you give appropriate credit to the original author(s) and the source, provide a link to the Creative Commons licence, and indicate if changes were made. The images or other third party material in this article are included in the article's Creative Commons licence, unless indicated otherwise in a credit line to the material. If material is not included in the article's Creative Commons licence and your intended use is not permitted by statutory regulation or exceeds the permitted use, you will need to obtain permission directly from the copyright holder. To view a copy of this licence, visit http://creativecommons.org/licenses/by/4.0/.

(C) The Author(s) 2021 